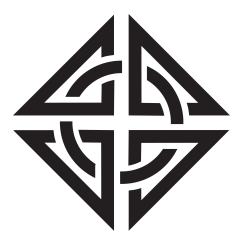

SCIENTIA
Sharif University of Technology

Scientia Iranica

Transactions E: Industrial Engineering

http://scientiairanica.sharif.edu

\title{
Coexistent scheduling in the tandem flow path configuration of a flexible manufacturing system by using an advanced grey wolf optimizer
}

\author{
V.K. Chawla ${ }^{a, *}$ A.K. Chanda ${ }^{b}$, S. Angra ${ }^{c}$, and A. Bonyadi ${ }^{d}$ \\ a. Department of Mechanical and Automation Engineering, Indira Gandhi Delhi Technical University for Women, Delhi, India. \\ b. Department of Mechanical and Automation Engineering, G.B. Pant Engineering College, Okhla, Delhi, India. \\ c. Department of Mechanical Engineering, The National Institutes of Technology, Kurukshetra, Haryana, India. \\ d. Department of Progress Engineering, Iran University of Science and Technology, Tehran, Iran.
}

Received 1 August 2019; received in revised form 27 August 2020; accepted 9 November 2020

\section{KEYWORDS}

Advanced grey wolf optimization;

Coexistent scheduling;

Flexible

manufacturing

system;

Material handling robots;

Tandem flow path configurations.

\begin{abstract}
The use of Material Handling Robots (MHRs) for efficient material handling operations in the Flexible Manufacturing Systems (FMSs) has gained wide popularity and acceptability across the automated production industries. Coexistent scheduling between jobs and MHRs improves the overall efficiency of the FMSs, significantly. In the present study, the coexistent scheduling between the MHRs and the jobs under production in the FMS was carried out by using an Advanced Grey Wolf Optimization (AGWO) algorithm. The proposed FMS layout was made up of tandem flow path configurations for the movement of the MHRs. The FMS constituted six Flexible Manufacturing Cells (FMCs) partitioned in six zones and served by six MHRs deployed in each partitioned zone for efficient material handling operations. To develop the coexistent schedule between MHRs and jobs, a combined objective function was formulated by incorporating two diverging objectives and solved by using the AGWO algorithm. The combined objective function for coexistent production scheduling in FMS, operating with 19 Work Centers (WCs) and six MHRs to produce 36 and 66 types of jobs in different batch production quantities, will be reported in the paper.

(C) 2022 Sharif University of Technology. All rights reserved.
\end{abstract}

\section{Introduction and related work}

The present era of circular economy calls for the production of jobs with customized design features and a small production cycle. This trend has triggered the requirement for product diversity and a varying quantity in the production of jobs. The use of a Flexible Manufacturing System (FMS) is the best alternative

*. Corresponding author.

E-mail addresses: vivekchawla@igdtuw.ac.in (V.K.

Chawla); akcped@yahoo.com (A.K. Chanda);

angrasurjit@yahoo.com (S. Angra); Bonyadi@iust.ac.ir (A. Bonyadi)

doi: $10.24200 /$ sci.2020.54152.3618 for catering to the diverse production requirements according to customer expectations. The production of jobs in the FMS is carried out under a highly uncertain and stochastic environment. The FMS can be described as a combination of different automated systems working together to achieve a common objective of production requirements. The different automated systems, which are observed to be working together in the FMS, can be classified as Automatic Guided Vehicles (AGVs) or Material Handling Robots (MHRs), conveyor systems, Work Centers (WCs), machining centers, inspection centers, assembly robots, welding robots, painting robots, packaging centers, and Automatic Storage and Retrieval Systems (ASRS), etc. The aforementioned automated systems are integrated 
via computers to work together on a common platform within the FMS facility. Different automated systems work in an FMS. Therefore, efficient scheduling to harness their best utilization is highly imperative.

Coexistent scheduling between different automated systems working in an FMS is observed to be more efficient than the conventional scheduling between different FMS resources Mohapatra et al. [1]. Coexistent scheduling in an FMS can be described as the development of schedules for more than one automated system, simultaneously, in order to execute the required operations on a combination of different jobs in the most efficient manner in a certain time frame [2-5]. In the process of coexistent scheduling, the schedule of one resource is given as input to the second resource so that the output schedule of the second resource is developed in concurrence with the first resource. Therefore, the coexistent schedule achieves the production requirements in the best possible manner $[2,5,6]$. In the FMS, an efficient coexistent schedule can be developed between jobs under production on different WCs and different autonomous MHRs, which are used for transferring the jobs to different WCs within the FMS facility. The coexistent scheduling between jobs and MHRs ensures the best utilization of all the WCs and the MHRs in the FMS [2,6,7].

The jobs are usually transferred by using an autonomous MHR, which moves on a flow path configuration within the FMS facility and transfers the jobs from one location to another one. Different types of flow path configurations can be designed in an FMS. The flow path configurations in an FMS can be broadly classified as a conventional, singleloop, or tandem [8-11]. The conventional flow path configurations offer connectivity to all WCs through a single flow path as portrayed in Figure 1. The conventional flow path configurations may have certain junctions and intersection points to meet the FMS requirements. The conventional flow path configuration is one of the most basic and common types of flow path configurations and found applicability in the early era of FMS installations. The single-loop

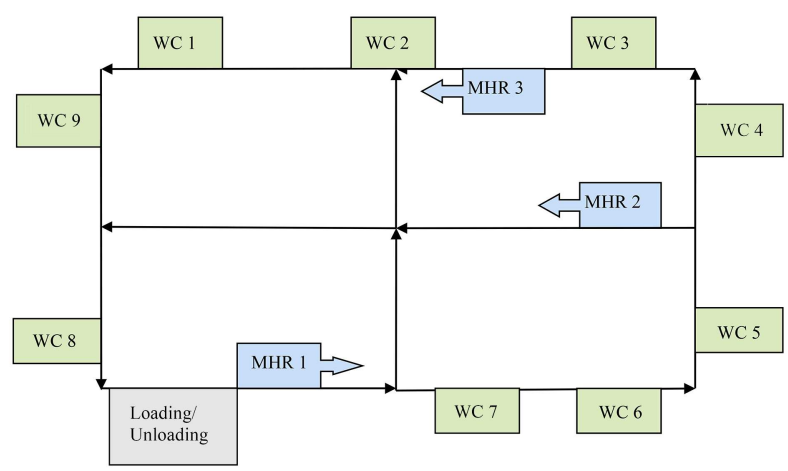

Figure 1. Conventional flow path configuration.

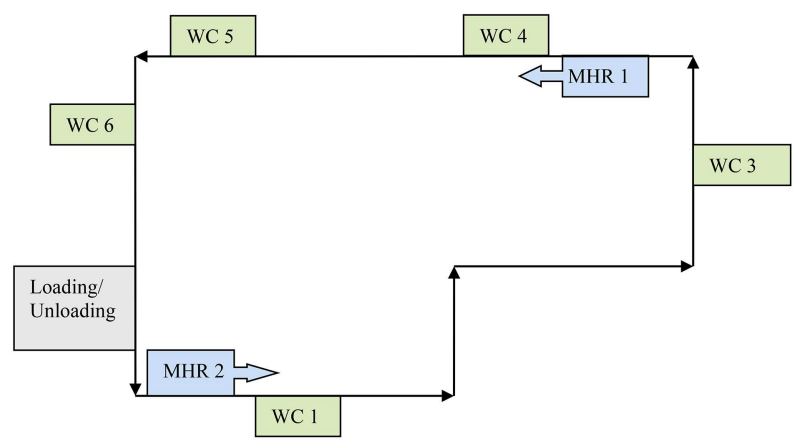

Figure 2. Single-loop flow path configuration.

flow path configuration, as shown in Figure 2, is an advancement of the conventional flow path configuration. In the single-loop flow path configuration, all WCs are connected to form a continuous loop and the MHRs travel in only one loop to transfer jobs from one location to another. The design of single-loop flow path configuration provides the opportunity to prevent intersection points and therefore, the probability of deadlock or collision between two or more MHRs is negligible.

The tandem flow path configuration was proposed by Bozer and Srinivasan [8] for efficient material transfer operations in an FMS. A tandem flow path configuration is portrayed in Figure 3 in which the FMS facility is divided into more than one zonal area. To each zonal area is allocated one MHR and some WCs. Each zone is also provided with one Transfer Point (TP) to transfer jobs from one zone to another by the moving MHRs. As one MHR is deployed in each zone, there are no chances of any deadlock or collision between moving MHRs in the FMS. This design of the tandem flow path configuration considerably increases the efficiency of a Material Handling System (MHS).

Bozer and Srinivasan [8] developed analytical models to find the throughput of an FMS operating in a tandem flow path configuration. Bozer and Srinivasan [9] introduced non-overlapping zonal areas for carrying out the material transfers by MHRs within the FMS facility. The authors used the First Encounter Fist Served (FEFS) dispatching rule for efficient material transfer operations.

\subsection{FMS scheduling}

FMS scheduling can be defined as the process of allocation of resources in an FMS to achieve production objectives in a specific period. An efficient production schedule yields high throughput and low makespan in the FMS. In an FMS, different production tasks are performed, namely jobs assembly, material transfers by MHRs from one point to another, quality inspection of jobs, machining of jobs, welding of jobs, packaging of jobs, etc. Chawla et al. [4,12], Udhayakumar and Kumanan [6,13], Angra et al. [10]. The optimum 


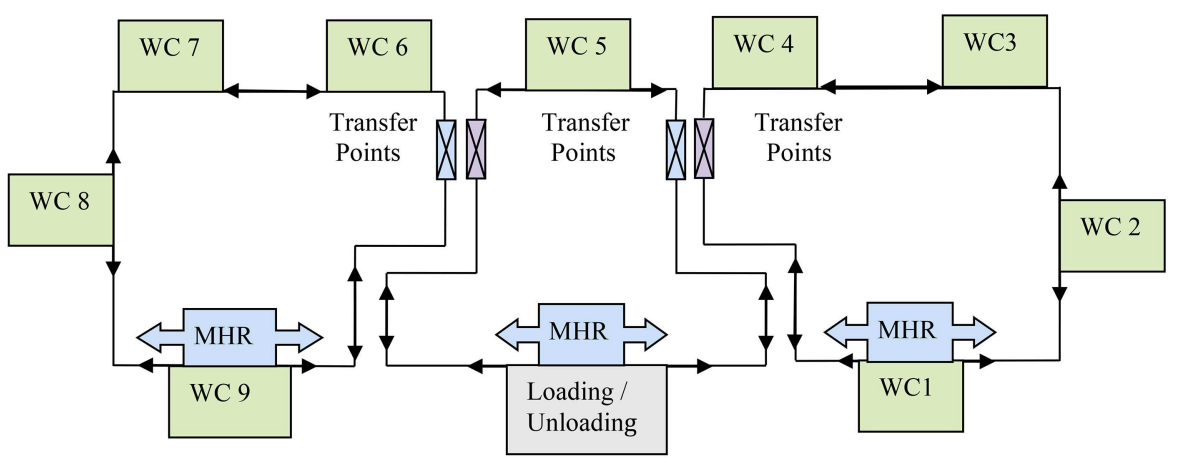

Figure 3. Tandem flow path configuration.

schedule for an FMS can be developed by synchronized operations of different FMS components.

The MHRs can be efficiently used for different material transfer requirements in an FMS or Flexible Manufacturing Cell (FMC) facility [2,4,6,13-19]. The synchronized scheduling issues in an FMS commonly require multi-objective decision making and call for solutions to combinatorial optimization scheduling problems in FMSs [20-28]. Commonly, scheduling objectives for an MHS are to minimize the transport time of MHRs, reduce material transfer distance, and optimize the MHRs fleet.

\subsection{Coexistent scheduling}

The coexistent scheduling problems are generally formulated by considering the scheduling of more than one resource, simultaneously. The WC and MHRs production scheduling issues are also considered, simultaneously [18,29-31]. Solving coexistent scheduling issues is always better than sequential scheduling optimization. The benefits of coexistent scheduling in comparison with sequential scheduling are lower error rate, search for optimum solutions in a larger search space, higher quality solutions, and reduced production time or make-span of jobs [32]. However, by formulating the scheduling issues of MHRs and FMS, simultaneously, the scheduling problem becomes uncertain and the solution procedures by the exact methods or analytical methods are found to be highly time-consuming and complex. To avoid complexity and time consumption of the solutions to dynamic scheduling issues, the heuristic approaches are preferred. The heuristic methods have proven capable of solving the two scheduling problems of WC and MHRs, simultaneously, until achieving the optimum results $[2,11,33-36]$.

The integrated or coexistent scheduling problem is one of the very typical problems to solve. A large set of scheduling options can be available for different FMS components, for example, schedule for different machining operations, schedule for WCs, schedule for tools, schedule for jobs, etc. $[37,38]$. A wide range of scheduling options can be observed when alternate work-centers are capable of similar machining operations on given jobs. Furthermore, other factors such as multipurpose tools, flexibility in the selection of sequences, etc. all provide a large set of options among which one can look for the best ones. Therefore, to find the best scheduling options, the constraints of any specific FMS must be observed so that an optimum scheduling system can be implemented $[33,35,39-$ 41].

The coexistent scheduling among the machines, MHRs, layout, AS/RS, tools, etc. in an FMS can yield high throughput, low makespan, low mean flow time, high WC utilization, high productivity, and low cost per unit produced in the FMS operations. The coexistent scheduling of machines and MHRs in an FMS can also offer a simultaneous reduction in the distance travelled and the number of backtrackings performed by the MHR in the FMS configuration. This, in turn, can also increase FMS production rate and WC utilization and reduce mean flow time [2,7,33-35]. Therefore, it becomes highly imperative to consider and carry out coexistent scheduling among machines, MHSs, and FMS configuration. With these said, it can be stated that the role of integrated scheduling in an FMS is highly significant from the economy and profitability points of view in manufacturing. Jerald et al. [34] used an Adaptive Genetic Algorithm (AGA) for the simultaneous scheduling of jobs and AGVs. They attempted to minimize the production idle time and maximize the FMS utilization. The authors compared the results of the AGA with a Genetic Algorithm (GA) for the same set of problems and found AGA superior to the GA. A hybrid method for the solution to AGVs routing and dispatching in an FMS was proposed by Corréa et al. [37]. They solved a multi-objective problem for simultaneous routing, dispatching, and task assignment of AGVs by dividing a master scheduling issue into sub-scheduling issues and then, solved it by using mixed-integer programming. Gamberi et al. [39] proposed an integrated flow model by considering the material handling activities of an in- 
dustrial facility operating with the maximum capacity. The proposed approach identified the production space needed, material transfer requirements, buffer capacity, due time, and economic evaluation of the material transfer operations in the facility layout. Nishi et al. [42] addressed multi-objective integrated scheduling and routing issues of AGVs for minimizing job tardiness. The authors divided the problem into a master problem and a sub-problem and solved them by the Lagrangian and lower bound, respectively. The proposed approach was validated through computational experiments. Chaudhry et al. [3] used a spreadsheetbased GA for solving an integrated scheduling issue between WCs and AGVs in the FMS facility. They focused on minimizing the make-span of jobs in the production facility. The results obtained from the proposed method were found effective. The integration of production schedule and material handling schedule to simultaneously minimize AGV distance travel and backtracking by using different heuristic algorithms has been proposed by Haq et al. [2], Udhayakumar and Kumanan [6], and Chawla et al. [7,14]. Nageswara et al. [43] used a hybrid meta-heuristic algorithm to simultaneously schedule WCs and AGVs in an FMS. The objective was to find the optimum sequence for low make-span and AGV scheduling for the production of 10 jobs in four different layouts.

Umar et al. [44] proposed a hybrid GA to solve an integrated scheduling issue of $\mathrm{AGVs}$ for minimizing the AGVs transport time, jobs production time, job delay, and production cost due to delay in the FMS. The authors formulated a multi-objective scheduling problem and solved it by using the fuzzy logic. Mallikarjuna et al. [45] used GA and Simulated Annealing (SA) to find an optimum guide path of AGVs for their simultaneous scheduling in the FMS. The simultaneous uni-directional guide path design and machine center scheduling issues were addressed by Zheng et al. [46]. The authors mentioned that the guide path design and AGV scheduling were interdependent. They applied a hybrid metaheuristic in order to minimize the makespan of jobs in the manufacturing facility. The integrated scheduling approach was highly efficient in the scheduling of small-size and medium-size production problems. However, in case of large-size scheduling issues, the sequential scheduling approach was found better than the integrated scheduling approach [32].

From the literature review, it is observed that several studies for integrated scheduling between MHRs and FMS have been performed by using different heuristic methods. However, coexistent scheduling between WCs and MHRs functioning in an FMS with tandem configuration has not been addressed in the literature. Therefore, a research gap regarding the coexistent scheduling by using an Advanced Grey
Wolf Optimization (AGWO) algorithm between the MHRs and different WCs functioning in an FMS with tandem configurations is evident. To fill in the aforesaid research gap, this paper aims to perform the coexistent scheduling between six partitioned zones of FMCs and six MHRs operating in an FMS with tandem configuration.

\section{Problem description}

In this paper, the FMS under consideration works in a tandem flow path configuration. The FMS facility is divided into six partitioned zones forming six FMCs. Each zone or FMC is deployed with a different number of WCs and two TPs for effective material transfer from one FMC to another one. Since the FMS has a tandem flow path configuration, each FMC is equipped with one dedicated MHR, which is responsible for transferring jobs in a raw, in semifinished or finished state from one FMC to another via the TPs within the entire FMS facility. The MHR is autonomous and free to move, carry, and transfer the jobs within the FMC. Two different loading and unloading centers can be used for entry and exit of jobs in the FMS. The pictorial representation of the FMS model under consideration is portrayed in the figure illustrating the tandem FMS configuration. Different types of computer-controlled WCs are installed to operate in each FMC. For example, FMC 1 constitutes three different types of WCs, FMC 2 constitutes four different types of WCs, FMC 3 constitutes five different types of WCs, FMC 4 and FMC 5 constitute two WCs, and FMC 6 constitutes three different types of WCs. To perform different machining operations, the WCs are provided with sufficient tools in their tool magazines.

The following assumptions are made for the functioning of the FMS.

1. The jobs are produced on the WCs working in the FMS having tandem configuration;

2. All WCs, MHRs, and other FMS equipment are $100 \%$ reliable and free from any kind of failure;

3. The jobs under production are subject to a cost in case of delay in their production operations;

4. The WCs are independent and a specific time is required for the completion of a production sequence;

5. If any specific WC is observed to be free in a specific FMC for a required production operation, then the job under production must be transferred to the free $\mathrm{WC}$ in the respective FMC without any delay;

6. All MHRs in the FMS have the same specifications and operating ranges; 
7. There is no deadlock or conflict in the movement of MHRs when transferring jobs within the FMS facility.

\subsection{Mathematical model and objective function}

In general, the overall objective of the production facility is to maintain high efficiency, i.e., high production rate with the lowest investment of funds in resources. In addition to high efficiency, the time vesting in the completion of different production operations, i.e., make-span as well as delay in the allocation of jobs to different resources of WCs, must also be minimized. WC utilization, MHRs utilization, production lead time, job tardiness, the percentage of tardiness, etc. can also be considered as prominent performance measures that can be used for analyzing the performance or efficiency of any production facility. The utilization of WCs can reflect the efficiency of the production facility, i.e., if the WCs are busy all the time, then their utilization will be high. One way to keep all WCs always busy is to implement the best scheduling strategies for all the under-production jobs in the FMS. Efficient scheduling of jobs also plays a pivotal role in minimizing delays in the production of jobs in the FMS. This further minimizes the penalty cost associated with the production delay.

Maximum involvement of WCs in the production operations also reflects more free time available to the MHRs, i.e., less utilization of MHRs. Less utilization of MHRs also reflects high utilization of WCs and minimum job delays. The coexistent scheduling between jobs and MHRs can minimize the production time of jobs and minimize the idle time of MHRs, significantly. Hence, in this paper, the coexistent scheduling between jobs and MHRs is proposed to achieve the Combined Objective Function (COF) of maximizing the WCs utilization and minimizing the cost associated with the production delay. The aforesaid objective function is mathematically formulated as given below:

$$
\begin{aligned}
& \text { Minimize objective function }=w_{1}\left[\frac{X_{c}}{M C O D}\right] \\
& \quad+w_{2}\left[\frac{I_{t}}{T_{t c}}\right] \\
& X_{c}=\sum_{i}\left(P T_{i}-T D_{i}\right) \times D C U_{i} \times S O B_{i}, \\
& P T_{i}=\text { Job } i(\text { production time })+\left(M H R_{t}\right), \\
& I_{t}=\sum_{j} W C_{i t}, \\
& W C_{i t}=I_{t}-\sum_{i} P T_{i j},
\end{aligned}
$$

where:

$\begin{array}{ll}w_{1} & \begin{array}{l}0.6 \text { weight attribute to minimize delay } \\ \text { cost }\end{array} \\ w_{2} & \begin{array}{l}0.4 \text { weight attribute to minimize WCs } \\ \text { idle time }\end{array} \\ X_{c} & \text { Total cost of delay } \\ M C O D & \text { Maximum Cost Of Delay } \\ I_{t} & \text { WCs idle time } \\ T_{t c} & \text { WCs total idle time } \\ i & \text { Job serial number } \\ j & \text { Work center serial number } \\ M H R_{t} & \text { Job transfer time taken by MHR } \\ T D_{i} & \text { Target date for production of job } i \\ D C U_{i} & \text { Delay cost per unit of job } i \\ S O B_{i} & \text { Size of a batch for job } i \\ P T_{i j} & \text { Time spent on the production of the } \\ & \text { ith job on the } j \text { th WC }\end{array}$

\section{The proposed GWO algorithm}

A GWO algorithm is a heuristic procedure to find optimized solutions to different problems. The GWO algorithm adopts the natural hunting behavior of grey wolfs for yielding the optimized results Mirjalili et al. $[47,48]$ and Bozorg-Haddad [49]. Grey wolves perform the hunting operation in groups of minimum four wolves, in which they initially search and target their prey, then they encircle it, after that they attack it, and at last, they hunt down the target prey. The GWO algorithm also works on the aforementioned intelligent hunting mechanism of grey wolves for finding the optimum solutions, in which a leadership hierarchy of wolves is developed. A group of four wolves termed as alpha $(\alpha)$, beta $(\beta)$, delta $(\Delta)$, and omega $(\omega)$ are considered in the algorithm. The leadership hierarchy between the wolves is always followed. The $\alpha$ wolf is the senior-most and always remains in the commanding position. The $\alpha$ wolf is responsible to make decisions about other wolves in the group. The $\beta$ wolf is placed on the second-best position in the group, considered as the next to the $\alpha$ wolf. Thereafter, the $\Delta$ and $\omega$ wolves are placed, respectively, i.e., the $\omega$ wolf has the lowest position in the hierarchy of the group. Therefore, the $\omega$ wolf follows the orders and directions as given by the $\alpha$, $\beta$, and $\Delta$ wolves, respectively. As the algorithm starts, the quantity and position of wolves are initialized. The fitness function for each wolf is defined as per its value, starting from the best one, i.e., the positioning of the wolves is alpha $(\alpha)$, beta $(\beta)$, delta $(\Delta)$, and then omega $(\omega)$, respectively. In the algorithm, $\vec{Z} p(t)$ represents prey position and $\vec{Z}(t)$ the position of a wolf during an iteration run. The encircling characteristic of grey wolves is exhibited by the following equations: 


$$
\begin{aligned}
& \vec{Y}=|\vec{K} \cdot \vec{Z} p(t)-\vec{Z}(t)|, \\
& \vec{Z}(t+1)=\vec{Z} p(t)-\vec{O} \cdot \vec{Y}, \\
& \vec{O}=2 \vec{a} \times \vec{r} 1-\vec{a}, \\
& \vec{K}=2 \vec{r} 2,
\end{aligned}
$$

where:

$t \quad$ Number of iterations,

$\vec{O}, \vec{K} \quad$ Coefficient vectors of the bootstrap program,

$\vec{Z} p \quad$ Position of prey,

$\vec{a} \quad$ Linear decrease in vector set from 2 to 0 during an iteration,

$\vec{r} 1, \vec{r} 2 \quad$ Randomized vectors in $[0,1]$.

The grey wolf positioned at $(x, y)$ keeps moving and its location is updated based on the location of the prey, i.e., $\left(x^{\prime}, y^{\prime}\right)$. The vectors are controlled to define and update the location of the best agent based on the location of prey. During the simulation run of the algorithm, the magnitude of $\vec{a}$ keeps on decreasing with the reduction in the fluctuation rate $\vec{O}$. The alpha $(\alpha)$, beta $(\beta)$, and delta $(\Delta)$ wolves store the position of the prey and based on their information, the three best solutions achieved are stored within the algorithm. The omega $(\omega)$, as the fourth agent, also updates its location in the simulation run of the algorithm with regard to the best solution achieved within the search space. An estimate of the location of the prey is found by the alpha $(\alpha)$, beta $(\beta)$, and delta $(\Delta)$, which is used to update the location of omega $(\omega)$ around it. The following equations represent the modeling of the GWO algorithm:

$$
\begin{aligned}
& \vec{Y} \alpha=|\vec{K} 1 \cdot \vec{X} \alpha-\vec{X}|, \\
& \vec{Y} \beta=|\vec{K} 2 \cdot \vec{X} \beta-\vec{X}|, \\
& \vec{Y} \Delta=|\vec{K} 3 \cdot \vec{X} \Delta-\vec{X}|, \\
& \vec{X} 1=\vec{X} \alpha-O 1 \cdot(\vec{Y} \alpha), \\
& \vec{X} 2=\vec{X} \beta-O 2 \cdot(\vec{Y} \beta), \\
& \vec{X} 3=\vec{X} \Delta-O 3 \cdot(\vec{Y} \Delta), \\
& \vec{X}(t+1)=\frac{\vec{X} 1+\vec{X} 2+\vec{X} 3}{3} .
\end{aligned}
$$

\subsection{AGWO algorithm for coexistent scheduling between jobs and MHRs}

To perform coexistent scheduling between jobs and MHRs, the GWO algorithm is used with some modifications. The steps for the application of the AGWO algorithm are expressed below and the pseudo-code is shown in Figure 4.

Step 1: Population initialization. Let $Q_{\max }$ be the number of wolves in the group and $Z p_{(t)}$ and $Z i_{(t)}$ represent the location of the grey wolf " $i$ " and prey " $p$ " such that $i=1,2,3,4, \cdots, Q_{\max }$, at " $t$ " iterations in the algorithm. In the beginning, a randomized value between 0 to 1 is given to the schedule jobs that are to be produced in all FMCs using MHRs. Thereafter, to find the job schedule, a Top Position Value (TPV) order is used to schedule the jobs under production in the FMS. One can refer to Table 1 as an instance for an FMS with 10 jobs under production, each given some randomized value. The FMC and MHRs job schedules are based on the assigned TPV, which can be considered as $\psi=(3,5,10,8,2,7,1,4,6$, and 9$)$. In this step, the wolves represent the FMCs and MHRs represent the job production sequence.

Step 2: Wolf sequencing. The solution observed in Step 1 is found by randomly determining a TPV order. To improvise the schedule of FMCs and MHRs, the NEH algorithm developed by Nawaz et al. [50] is used. The results of the NEH algorithm are further modified after assigning 1 to the job in the first position in the FMC and MHR schedule, $1-1 / n$ to the second job, and $1-2 / n$ to the third job in the schedule. The procedure is followed up to the last job in the production.

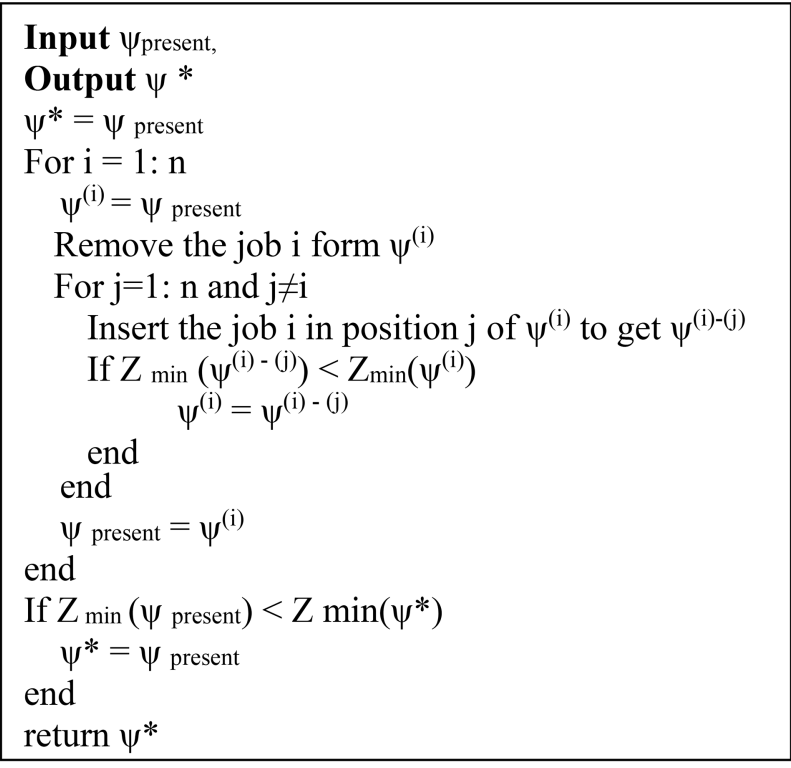

Figure 4. Pseudo-code for the local search algorithm. 
Table 1. Randomized values of job scheduling for the FMC and MHR.

\begin{tabular}{lcccccccccc}
\hline FMC and MHR job $\boldsymbol{i}$ & $\mathbf{1}$ & $\mathbf{2}$ & $\mathbf{3}$ & $\mathbf{4}$ & $\mathbf{5}$ & $\mathbf{6}$ & $\mathbf{7}$ & $\mathbf{8}$ & $\mathbf{9}$ & $\mathbf{1 0}$ \\
Randomized value & 0.61 & 0.70 & 0.85 & 0.56 & 0.82 & 0.48 & 0.68 & 0.76 & 0.43 & 0.80 \\
\hline
\end{tabular}

Step 3: Fitness function evaluation of the $\alpha, \beta$, and $\Delta$ wolves. The fitness of the solutions (job schedule) is calculated by Eqs. (1) and (17):

$$
F(V i)=\mu-T(V i) \text { for } i=1,2, \cdots, Q_{\max },
$$

where $\mu$ is the highest positive value and the value of $T\left(V_{i}\right)$ can be found from Eq. (1).

Step 4: Sorting of the $\alpha, \beta$, and $\Delta$ wolves. The job scheduling is done according to the fitness functions achieved, i.e., $X_{\alpha}, X_{\beta}$, and $X_{\Delta}$ considered as the first, second, and third jobs, respectively. Thereafter, to find the best positions for $X_{\alpha}, X_{\beta}$, and $X_{\Delta}$, respectively, a local search procedure is followed for up to 10 iterations, after which the best coexistent schedule is selected for the production of the jobs in the FMS. The local search procedure is illustrated in the form of pseudocode in Figure 4. In case any difference is observed in the old fitness value of a solutions in comparison with the new fitness values of solutions, the order $X_{\alpha}, X_{\beta}$, and $X_{\Delta}$ is stored and updated.

Step 5: Using Eq. (16) to update the location of the $\omega$ wolf.

Step 6: Following Step 2 to evaluate the fitness of each wolf after their movements.

Step 7: Identifying the new values of $\alpha, \beta$, and $\Delta$ to find and update the new positions of the $\alpha, \beta$, and $\Delta$ wolves.
In the advanced GWO algorithm, Steps 5 to 7 are repeated until the required objective is achieved.

\subsection{Numerical Example 1}

The proposed FMS facility constitutes six FMCs operating in a tandem flow path configuration as given in Figure 5. In each FMC, the MHR is deployed to transfer materials or jobs from one FMC to another. The jobs are transferred via the TPs installed in each FMC. The MHR is autonomous and has bidirectional movement capability in the FMC. The proposed FMS is equipped with two loading and unloading centers for passing in and out jobs in the FMS facility. The FMCs are equipped with highly automated WCs of versatile specifications and capable of producing jobs with high accuracy and precision. In the proposed FMS facility, FMC 1 has three different WCs, FMC 2 is run with four WCs, FMC 3 is installed with five different types of WCs, FMC 4, and FMC 5 work with 2 WCs, and FMC 6 is equipped with three different WCs. Hence, in total, the FMS facility is equipped with 19 WCs to produce different types of jobs in batch production quantities.

In Numerical Example 1, 36 jobs are to be produced in the proposed FMS facility as shown in Figure 5. The detailed production data for 36 jobs are provided in Table 2. In Table 2, the production sequence for each job in the required FMC, information on their WC, and the production time are provided. Similarly, the production batch quantity, target days

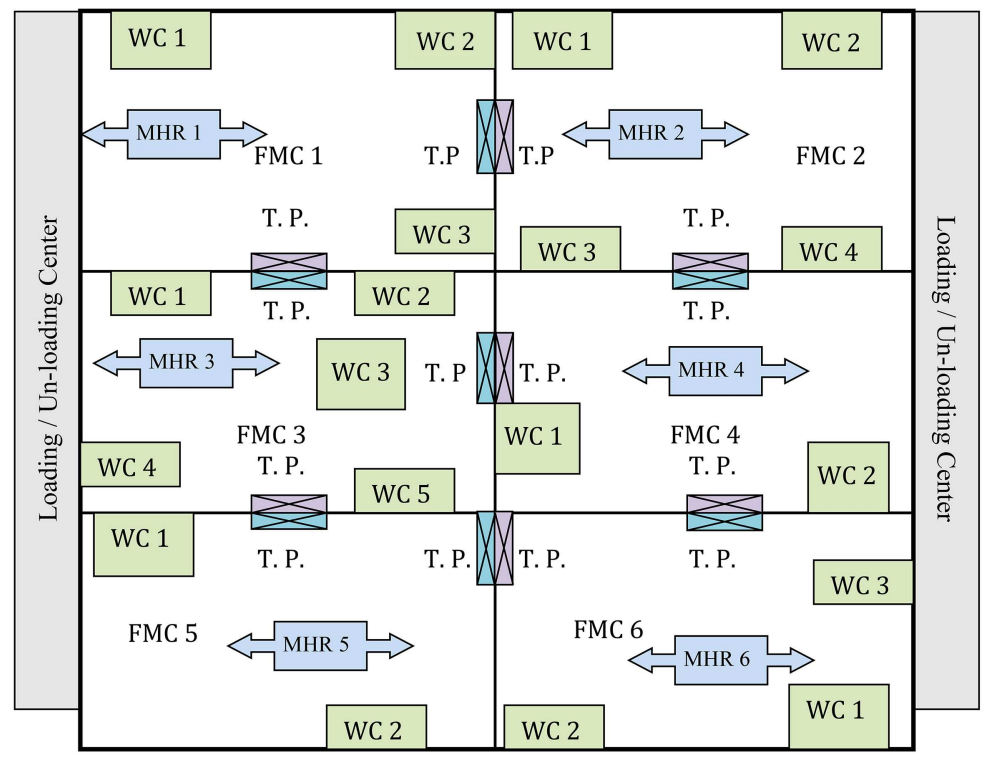

Figure 5. Tandem FMS configuration. 
Table 2. Production data for different jobs in the FMS for Numerical Example 1.

\begin{tabular}{|c|c|c|c|c|}
\hline $\begin{array}{c}\text { Job } \\
\text { number }\end{array}$ & $\begin{array}{l}\text { Job production sequence-FMC no. } \\
\text { (work-center number and production time) }\end{array}$ & $\begin{array}{c}\text { Target } \\
\text { date in } \\
\text { days }\end{array}$ & $\begin{array}{l}\text { Quantity } \\
\text { of job }\end{array}$ & $\begin{array}{l}\text { Cost of delay } \\
\text { Rs/Units/Day }\end{array}$ \\
\hline 1 & FMC $1(2,2)$, FMC $2(1,1)$, FMC $3(3,2)$, FMC $4(2,1)$, FMC $5(1,2)$ & 19 & 250 & 1.00 \\
\hline 2 & FMC $3(2,3)$, FMC $4(2,2)$, FMC $1(3,2)$ & 16 & 300 & 1.00 \\
\hline 3 & FMC $1(3,2)$, FMC $2(3,1)$, FMC $4(1,1)$, FMC $5(2,1)$ & 12 & 600 & 1.00 \\
\hline 4 & $\operatorname{FMC} 5(1,2), \operatorname{FMC} 6(3,1), \operatorname{FMC} 4(2,2), \operatorname{FMC} 3(4,1)$ & 22 & 850 & 2.00 \\
\hline 5 & $\begin{array}{l}\text { FMC } 1(3,1), \text { FMC } 2(4,1), \operatorname{FMC} 3(5,2), \text { FMC } 4(2,1), \operatorname{FMC} 5(1,1), \\
\text { FMC } 6(2,2)\end{array}$ & 13 & 200 & 1.00 \\
\hline 6 & FMC $2(3,2)$, FMC $4(2,1)$, FMC $3(4,2)$ & 17 & 900 & 2.00 \\
\hline 7 & $\operatorname{FMC} 3(3,2), \operatorname{FMC} 5(1,1), \operatorname{FMC} 6(2,1), \operatorname{FMC} 1(3,1)$ & 19 & 300 & 1.00 \\
\hline 8 & $\begin{array}{l}\text { FMC } 1(3,1), \text { FMC } 2(4,1), \text { FMC } 3(5,2), \text { FMC } 4(2,1), \text { FMC } 5(1,1), \\
\text { FMC } 6(2,2)\end{array}$ & 14 & 500 & 1.00 \\
\hline 9 & FMC 2(4,1), FMC 1(3,1), FMC $3(2,2)$ & 2 & 150 & 0.00 \\
\hline 10 & FMC $1(3,1)$, FMC $2(4,2)$ & 15 & 300 & 2.00 \\
\hline 11 & $\operatorname{FMC} 1(1,3), \operatorname{FMC} 2(2,3), \operatorname{FMC} 3(5,1), \operatorname{FMC} 4(1,1), \operatorname{FMC} 5(1,2)$ & 3 & 300 & 1.00 \\
\hline 12 & $\begin{array}{l}\text { FMC } 1(1,1), \operatorname{FMC} 2(3,2), \operatorname{FMC} 3(3,3), \text { FMC } 4(1,2), \operatorname{FMC} 5(2,1) \text {, } \\
\text { FMC } 6(3,2)\end{array}$ & 11 & 900 & 3.00 \\
\hline 13 & FMC 5(2,1), FMC 4(1,2), FMC 6(2,3) & 22 & 800 & 4.00 \\
\hline 14 & FMC $4(2,1)$, FMC $3(4,1)$, FMC $2(3,1)$, FMC $1(3,2)$ & 16 & 750 & 3.00 \\
\hline 15 & $\operatorname{FMC} 3(4,2), \operatorname{FMC} 4(1,2), \operatorname{FMC} 1(2,1), \operatorname{FMC} 5(1,2)$ & 10 & 1000 & 5.00 \\
\hline 16 & FMC $1(1,1)$, FMC $2(3,2)$, FMC $3(5,2)$ & 23 & 800 & 3.00 \\
\hline 17 & $\begin{array}{l}\text { FMC } 1(3,1), \operatorname{FMC} 2(4,1), \operatorname{FMC} 3(5,2), \operatorname{FMC} 4(2,1), \operatorname{FMC} 5(1,1), \\
\operatorname{FMC} 6(2,2)\end{array}$ & 17 & 700 & 3.00 \\
\hline 18 & FMC 3(3,2), FMC 4(1,2), FMC5(1,1) & 21 & 400 & 6.00 \\
\hline 19 & $\operatorname{FMC} 1(3,1), \operatorname{FMC} 2(1,3), \operatorname{FMC} 3(5,2), \operatorname{FMC} 4(1,1)$ & 9 & 350 & 1.00 \\
\hline 20 & $\operatorname{FMC} 1(2,1), \operatorname{FMC} 2(3,2), \operatorname{FMC} 3(4,1), \operatorname{FMC} 4(1,1), \operatorname{FMC} 5(1,3)$ & 1 & 100 & 0.0 \\
\hline 21 & FMC $4(2,3)$, FMC $5(1,2)$ & 12 & 900 & 3.00 \\
\hline 22 & FMC $3(4,1)$, FMC $6(2,3)$ & 20 & 300 & 4.00 \\
\hline 23 & $\begin{array}{l}\text { FMC } 1(3,1), \text { FMC } 2(4,1), \operatorname{FMC} 3(5,2), \text { FMC } 4(2,1), \operatorname{FMC} 5(1,1), \\
\text { FMC } 6(2,2)\end{array}$ & 16 & 100 & 4.00 \\
\hline 24 & FMC $3(2,3)$, FMC $4(2,1)$, FMC $5(1,4)$, FMC $4(1,1)$ & 9 & 300 & 4.00 \\
\hline 25 & $\operatorname{FMC} 1(3,2), \operatorname{FMC} 2(4,1), \operatorname{FMC} 2(3,2)$ & 21 & 400 & 2.00 \\
\hline 26 & FMC $1(3,1)$, FMC $2(4,1)$, FMC $3(5,2)$ & 25 & 300 & 1.00 \\
\hline 27 & FMC $1(2,2)$, FMC $1(3,1)$ & 16 & 500 & 2.00 \\
\hline 28 & FMC $4(1,1)$, FMC $2(1,3)$, FMC $5(2,2)$, FMC $5(1,1)$, FMC $2(3,3)$ & 6 & 700 & 3.00 \\
\hline 29 & FMC $2(2,3)$, FMC $2(1,2)$, FMC $2(3,2)$, FMC $3(2,3)$ & 9 & 900 & 1.00 \\
\hline 30 & FMC $1(3,2)$, FMC $2(2,1)$, FMC $3(1,2)$ & 12 & 900 & 1.00 \\
\hline 31 & FMC $1(2,2)$, FMC $2(1,1)$, FMC $3(3,2)$ & 5 & 700 & 2.00 \\
\hline 32 & $\operatorname{FMC} 1(3,1), \operatorname{FMC} 2(4,1), \operatorname{FMC} 3(5,2)$ & 19 & 1000 & 3.00 \\
\hline 33 & FMC $1(3,1)$, FMC $2(3,2), \operatorname{FMC} 3(4,3)$, FMC $4(1,2)$, FMC $5(1,3)$ & 21 & 600 & 3.00 \\
\hline 34 & $\operatorname{FMC} 1(3,1), \operatorname{FMC} 2(1,3), \operatorname{FMC} 3(4,2), \operatorname{FMC} 3(1,3)$ & 16 & 800 & 6.00 \\
\hline 35 & FMC $1(3,1)$ & 2 & 200 & 0.0 \\
\hline 36 & $\begin{array}{l}\text { FMC } 1(3,1), \text { FMC } 2(4,1), \operatorname{FMC} 3(5,2), \text { FMC } 4(2,1), \text { FMC } 5(1,1) \text {, } \\
\text { FMC } 6(2,2)\end{array}$ & 11 & 900 & 2.00 \\
\hline
\end{tabular}

for production of each job, and the associated delay cost per unit per day are also given in Table 2 for the production of jobs.

\subsection{Numerical Example 2}

In Numerical Example 2, 66 jobs are to be produced by the proposed FMS facility, portrayed in Figure 5. The production data for the 66 jobs are mentioned in Table 3. Similar to Tables 2 and 3 provides information regarding the production sequence of jobs in the FMC and their WC number and production time.

\section{Results and discussions}

The FMS operating in tandem flow path configuration is considered for the coexistent scheduling between jobs and MHRs. The FMS facility constitutes six partitioned zones as the FMCs with several WCs for the production of different job combinations and one 
Table 3. Production data for different jobs in the FMS for Numerical Example 2.

\begin{tabular}{|c|c|c|c|c|}
\hline $\begin{array}{c}\text { Job } \\
\text { number }\end{array}$ & $\begin{array}{l}\text { Job production sequence-FMC no. } \\
\text { (work-center number and production time) }\end{array}$ & $\begin{array}{c}\text { Target } \\
\text { date in } \\
\text { days }\end{array}$ & $\begin{array}{l}\text { Quantity } \\
\text { of job }\end{array}$ & $\begin{array}{l}\text { Cost of delay } \\
\text { Rs/Units/Day }\end{array}$ \\
\hline 1 & FMC $3(4,3)$, FMC $4(2,2)$, FMC $5(1,3)$ & 15 & 400 & 2.00 \\
\hline 2 & FMC $2(3,2)$, FMC $3(4,5)$, FMC $4(1,3)$, FMC $1(3,1)$ & 18 & 200 & 1.00 \\
\hline 3 & FMC $1(1,3)$, FMC $2(2,4)$, FMC $3(4,2)$, FMC $4(1,3)$, FMC $5(1,3)$ & 14 & 500 & 1.00 \\
\hline 4 & FMC 2(4,2), FMC $3(3,3)$, FMC 4(1,3), FMC 5(2,3), FMC $6(2,4)$ & 21 & 600 & 1.00 \\
\hline 5 & FMC $1(3,2)$, FMC $2(2,3)$, FMC $3(3,4)$ & 10 & 150 & 2.00 \\
\hline 6 & FMC $2(2,3)$, FMC $3(5,4)$, FMC $4(1,3)$, FMC $5(1,5)$ & 12 & 700 & 3.00 \\
\hline 7 & FMC $3(2,4)$, FMC 5(1,3), FMC 6(2,4), FMC 1(1,4), FMC 2(1,4) & 12 & 500 & 1.00 \\
\hline 8 & FMC $1(2,4)$, FMC $2(3,4)$, FMC $3(3,5)$, FMC $4(1,4)$, FMC $5(2,2)$ & 9 & 600 & 2.00 \\
\hline 9 & FMC $1(3,2)$, FMC $2(3,4)$, FMC $3(4,5)$, FMC $4(1,3)$, FMC $5(1,2)$ & 7 & 250 & 1.00 \\
\hline 10 & FMC $1(1,3)$, FMC $2(3,5)$, FMC $3(4,3)$, FMC $4(2,3)$ & 4 & 100 & 1.00 \\
\hline 11 & FMC $1(2,4)$, FMC $2(3,2)$, FMC $3(5,4)$ & 2 & 400 & 1.00 \\
\hline 12 & FMC 1(3,2), FMC 2(4,1), FMC 3(2,4) & 9 & 600 & 2.00 \\
\hline 13 & FMC $1(2,3)$, FMC $2(1,3)$, FMC $3(1,4)$, FMC $4(1,3)$ & 11 & 700 & 5.00 \\
\hline 14 & $\begin{array}{l}\text { FMC } 1(2,5), \text { FMC } 2(1,3), \text { FMC } 3(2,4), \text { FMC } 4(1,3), \text { FMC } 5(2,3) \text {, } \\
\text { FMC } 6(3,5)\end{array}$ & 14 & 950 & 2.00 \\
\hline 15 & FMC $1(3,4)$, FMC $2(2,4)$, FMC $3(5,3)$ & 15 & 900 & 2.00 \\
\hline 16 & FMC $1(2,3)$, FMC $2(1,4)$, FMC $3(5,1)$, FMC $4(1,1)$ & 6 & 700 & 1.00 \\
\hline 17 & FMC $1(1,2)$, FMC $2(3,4)$, FMC $3(2,4)$, FMC $4(1,3)$, FMC $5(2,3)$ & 15 & 400 & 2.00 \\
\hline 18 & FMC $3(1,2)$, FMC $4(3,4)$, FMC $5(2,3)$, FMC $6(2,4)$ & 14 & 300 & 4.00 \\
\hline 19 & FMC $1(1,2)$, FMC $2(3,4)$, FMC $3(4,1)$, FMC $4(2,5)$, FMC $5(1,3)$ & 18 & 550 & 2.00 \\
\hline 20 & FMC $1(3,4)$, FMC 2(2,5) & 7 & 200 & 1.00 \\
\hline 21 & FMC $1(2,3)$, FMC $2(1,4), \operatorname{FMC} 3(3,4), \operatorname{FMC} 4(1,4)$, FMC $5(1,3)$ & 16 & 800 & 2.00 \\
\hline 22 & FMC $3(2,4)$, FMC $4(1,5)$, FMC $5(2,4)$ & 22 & 600 & 3.00 \\
\hline 23 & FMC $1(1,3)$, FMC $2(2,3)$, FMC $3(1,4)$, FMC $4(1,5)$ & 15 & 200 & 2.00 \\
\hline 24 & FMC $3(1,4)$, FMC 4(1,2), FMC 5(1,3) & 11 & 450 & 3.00 \\
\hline 25 & FMC 1(2,3), FMC 2(1,2), FMC 4(1,1) & 19 & 600 & 1.00 \\
\hline 26 & $\operatorname{FMC} 1(2,4), \operatorname{FMC} 2(1,3), \operatorname{FMC} 3(2,4)$, FMC $2(3,2)$ & 22 & 400 & 2.00 \\
\hline 27 & $\operatorname{FMC} 1(3,2), \operatorname{FMC} 1(2,3)$, FMC $3(1,3)$, FMC $4(2,4)$ & 18 & 200 & 1.00 \\
\hline 28 & $\mathrm{FMC} 1(2,4), \mathrm{FMC} 2(3,2), \mathrm{FMC} 3(4,5), \mathrm{FMC} 2(2,1), \mathrm{FMC} 5(2,1)$ & 9 & 400 & 2.00 \\
\hline 29 & FMC 2(3,4), FMC 2(1,3), FMC 2(4,1), FMC 3(1,4), FMC $3(5,3)$ & 6 & 600 & 2.00 \\
\hline 30 & FMC $1(2,4)$, FMC $2(1,4)$, FMC $3(2,3)$, FMC $5(1,4)$ & 7 & 1000 & 3.00 \\
\hline 31 & $\operatorname{FMC} 1(1,3), \operatorname{FMC} 2(2,4), \operatorname{FMC} 2(3,5), \operatorname{FMC} 3(2,3)$ & 15 & 800 & 4.00 \\
\hline 32 & $\operatorname{FMC} 1(2,4), \operatorname{FMC} 2(3,5), \operatorname{FMC} 3(2,4), \operatorname{FMC} 3(3,4)$ & 22 & 700 & 2.00 \\
\hline 33 & FMC $1(2,4)$, FMC $2(1,3)$, FMC $3(2,4)$ & 24 & 400 & 2.00 \\
\hline 34 & FMC $1(1,3)$, FMC $2(1,4)$, FMC $2(2,4)$, FMC $3(4,2)$, FMC $5(2,1)$ & 18 & 700 & 4.00 \\
\hline 35 & FMC 1(2,4), FMC 2(1,3) & 5 & 400 & 2.00 \\
\hline 36 & FMC $1(2,3)$, FMC $2(2,3)$, FMC $4(3,4)$, FMC $6(3,4)$ & 14 & 700 & 0.00 \\
\hline 37 & $\begin{array}{l}\operatorname{FMC} 1(1,2), \operatorname{FMC} 3(2,3), \operatorname{FMC} 3(2,4), \operatorname{FMC} 4(1,4), \operatorname{FMC} 4(2,3), \\
\operatorname{FMC} 5(1,1)\end{array}$ & 21 & 450 & 1.00 \\
\hline 38 & FMC $3(1,4)$, FMC $4(1,3)$, FMC $2(3,4)$ & 18 & 200 & 1.00 \\
\hline 39 & $\begin{array}{l}\text { FMC } 1(2,4), \text { FMC } 2(1,3), \text { FMC } 2(3,3), \text { FMC } 2(4,3), \text { FMC } 4(1,2), \\
\text { FMC } 5(2,3)\end{array}$ & 14 & 400 & 3.00 \\
\hline
\end{tabular}


Table 3. Production data for different jobs in the FMS for Numerical Example 2 (continued).

\begin{tabular}{|c|c|c|c|c|}
\hline $\begin{array}{c}\text { Job } \\
\text { number }\end{array}$ & $\begin{array}{c}\text { Job production sequence-FMC no. } \\
\text { (work-center number and production time) }\end{array}$ & $\begin{array}{c}\text { Target } \\
\text { date in } \\
\text { days }\end{array}$ & $\begin{array}{l}\text { Quantity } \\
\text { of job }\end{array}$ & $\begin{array}{l}\text { Cost of delay } \\
\text { Rs/Units/Day }\end{array}$ \\
\hline 40 & FMC $6(1,3)$, FMC $4(1,3)$, FMC $4(2,4)$, FMC $3(2,2)$ & 18 & 650 & 1.00 \\
\hline 41 & FMC 1(2,2), FMC 1(3,3), FMC 2(4,2), FMC 3(2,3), FMC 6(1,4), & 16 & 300 & 2.00 \\
\hline 42 & FMC 2(2,5), FMC 4(2,4), FMC 3(2,3) FMC 6(2,3), & 19 & 500 & 1.00 \\
\hline 43 & $\begin{array}{l}\text { FMC } 3(1,4), \text { FMC } 3(2,3), \text { FMC } 5(1,2), \text { FMC } 5(2,3), \text { FMC } 1(3,3) \text {, } \\
\text { FMC } 1(2,2)\end{array}$ & 21 & 400 & 2.00 \\
\hline 44 & FMC $1(1,4)$, FMC $2(3,2)$, FMC $2(1,4), \operatorname{FMC} 3(5,4)$ & 9 & 250 & 1.00 \\
\hline 45 & FMC $2(3,2)$, FMC $1(2,4)$, FMC $3(4,3)$, FMC $3(3,4)$ & 4 & 350 & 2.00 \\
\hline 46 & FMC $1(2,4)$, FMC $3(4,5)$, FMC $6(3,2)$ & 11 & 400 & 1.00 \\
\hline 47 & FMC 2(3,1), FMC $3(3,4)$ & 2 & 600 & 1.00 \\
\hline 48 & $\begin{array}{l}\text { FMC } 2(1,3), \text { FMC } 2(2,4), \text { FMC } 3(1,4), \text { FMC } 4(1,3), \text { FMC } 6(1,4) \text {, } \\
\text { FMC } 6(3,3)\end{array}$ & 9 & 400 & 0.00 \\
\hline 49 & FMC $4(1,2)$, FMC $5(1,3)$, FMC $4(2,23)$, FMC $6(1,4)$, FMC $6(3,2)$ & 11 & 400 & 2.00 \\
\hline 50 & FMC $1(1,3)$, FMC $2(3,2), \operatorname{FMC} 3(2,4), \operatorname{FMC} 3(3,3)$ & 14 & 450 & 2.00 \\
\hline 51 & FMC $3(1,3)$, FMC $4(2,3)$, FMC $4(1,3)$, FMC $1(1,4)$, FMC $6(2,3)$ & 9 & 900 & 3.00 \\
\hline 52 & FMC $1(2,3)$, FMC $2(1,3)$, FMC $2(3,4)$ FMC $3(3,4)$, FMC $5(1,1)$ & 15 & 500 & 4.00 \\
\hline 53 & FMC 1(2,3), FMC 1(1,4), FMC 2(1,3), FMC 4(1,3), FMC 5(2,3) & 23 & 400 & 1.00 \\
\hline 54 & FMC $3(4,1)$, FMC $4(2,3)$, FMC $5(1,2)$, FMC $5(2,2)$, FMC $6(1,3)$ & 24 & 700 & 4.00 \\
\hline 55 & $\operatorname{FMC} 1(3,3), \operatorname{FMC} 3(1,4), \operatorname{FMC} 3(2,3), \operatorname{FMC} 4(2,3)$ & 11 & 850 & 2.00 \\
\hline 56 & FMC $1(2,1)$, FMC $2(1,4)$, FMC $2(1,3)$, FMC $3(2,4)$, FMC $4(1,4)$ & 14 & 650 & 1.00 \\
\hline 57 & FMC $1(3,1)$, FMC $1(2,1)$, FMC $2(4,3)$, FMC $2(2,3)$ & 10 & 750 & 1.00 \\
\hline 58 & FMC $2(2,4)$, FMC $3(1,3)$, FMC $3(2,4)$, FMC $6(2,4)$ & 9 & 450 & 0.00 \\
\hline 59 & FMC $2(1,4)$, FMC $2(4,3)$, FMC $3(2,5)$, FMC $6(1,3)$ & 20 & 900 & 3.00 \\
\hline 60 & FMC $1(2,4)$, FMC $3(1,4)$, FMC $6(2,5)$ & 18 & 700 & 2.00 \\
\hline 61 & FMC $6(2,4)$, FMC $3(1,4)$, FMC $3(5,2)$ & 19 & 650 & 3.00 \\
\hline 62 & FMC $1(2,4)$, FMC $2(2,5)$, FMC $4(2,2)$ & 6 & 300 & 1.00 \\
\hline 63 & FMC $5(1,4)$, FMC $6(1,5)$, FMC $6(3,4)$ & 15 & 950 & 1.00 \\
\hline 64 & FMC $1(2,1)$, FMC $3(1,2)$, FMC $3(4,3)$, FMC $6(1,3)$, FMC $6(3,3)$ & 14 & 1000 & 1.00 \\
\hline 65 & FMC $2(3,2)$, FMC $2(2,4)$, FMC $4(1,2)$ & 12 & 600 & 2.00 \\
\hline 66 & FMC $1(2,1)$, FMC $3(2,3)$, FMC $3(1,2)$, FMC $5(2,1)$ & 11 & 700 & 1.00 \\
\hline
\end{tabular}

MHR for transferring the jobs from one WC to another. The job scheduling and MHRs scheduling are carried out simultaneously for efficient utilization of the FMS by minimizing a COF using an AGWO algorithm. The performance of the AGWO is observed to be dependent on the size of agents (wolves), number of iterations, and tuning of parameters.

In this work, an Intel (R) Core(TM) i5 computer processor was used for iterating the algorithm. The simulation was run in 120 iterations. A population size of 50 was considered. Parametric tuning of the algorithm was done with two randomized vectors at the level of $[0,1]$. The controlling vector decreased linearly by a vector set from 2 to 0 during the iterative process.

During the run of iterations, it was observed that the advanced AGWO yielded near optimal results before achieving the termination criteria. The job schedule for 25 samples was considered having a minimum COF and near optimal value as an outcome of the AGWO. The COFs of an advanced grey wolf optimizer for the solutions to two numerical Examples 1 and 2 are mentioned in Table 4 . The COF of the coexistent schedule for the production of 36 jobs was 0.30127 and for the production of 66 jobs was 0.32341 . The size of the FMS facility remained the same for the production of both job quantities, including six portioned zones, 19 automated WCs, and six MHRs in both production scenarios. From the results, it was evident that if the production load on the FMS facility increased to around twice, then the achieved COF by the AGWO would increase by 0.02214 , i.e., a rise of $7.34 \%$. This increase in COF value with an increase in production load reflected that the performance of the AGWO also changed to $7.34 \%$ if the production load was nearly doubled in the aforesaid FMS facility. 
Table 4. Combined objective function achieved by the advanced grey wolf optimizer.

\begin{tabular}{|c|c|c|c|c|}
\hline $\begin{array}{l}\text { Numerical } \\
\text { example }\end{array}$ & Algorithm & Job sequencing & MHR sequencing & $\begin{array}{l}\text { Combined } \\
\text { objective } \\
\text { function }\end{array}$ \\
\hline 1 & Advanced & $\begin{array}{l}21-11-16-22-31-36-09-02-13-17-18- \\
32-34-20-23-25-27-29-35-07-04-08- \\
15-19-12-01-05-06-14-24-28-33-30- \\
03-10-26\end{array}$ & $\begin{array}{l}5416162346235135514625 \\
1263343246651421536\end{array}$ & 0.30127 \\
\hline 2 & $\begin{array}{l}\text { grey wolf } \\
\text { optimizer }\end{array}$ & $\begin{array}{l}31-25-42-15-21-54-23-04-11-16-29- \\
51-60-10-26-37-20-05-13-28-44-57- \\
09-14-62-47-18-39-24-08-17-64-59- \\
38-32-46-01-50-65-12-03-27-43-52- \\
33-41-45-66-02-30-48-58-61-06-34- \\
55-22-56-19-07-49-35-63-53-40-36\end{array}$ & 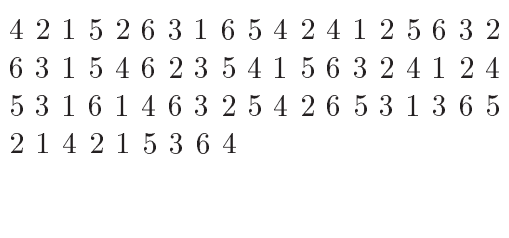 & 0.32341 \\
\hline
\end{tabular}

In addition, the AGWO appeared to be an effective algorithm for the development of coexistent production schedules between MHRs and WCs functioning in a tandem flow path FMS configuration. It was also observed that the job sequence output by the AGWO led to a lower penalty cost by maximum WC utilization in both numerical examples.

\section{Conclusion}

In this paper, the conventional Grey Wolf Optimization (GWO) algorithm was developed into an advanced grey wolf optimizer to perform coexistent scheduling between Material Handling Robots (MHRs) and Flexible Manufacturing Cells (FMCs) in an Flexible Manufacturing System (FMS) operating in tandem flow path configuration. Two opposite objectives were formulated as a combined objective function to minimize the production time of jobs as well as the idle time of the MHRs, simultaneously, by using the Advanced Grey Wolf Optimization (AGWO). To test the efficiency of the proposed approach, two numerical examples were solved for coexistent scheduling of 36 jobs and 66 jobs and MHRs in a tandem FMS configuration. From computational results, it was observed that the proposed method could be effectively employed to achieve the best coexistent scheduling decisions for the production of 36 jobs and 66 jobs. The conventional GWO was hybridized by using a local search procedure to sequence and sort the wolves in the algorithm. The proposed coexistent scheduling method minimized the values of the combined objective function and led to improved results overall for the best utilization of MHRs and FMCs. However, it was observed that if the production load on the FMS facility increased almost twice, then the combined objective function achieved by the AGWO would increase by $7.34 \%$.

The future research work may be directed to solving coexistent scheduling issues by advanced evo- lutionary algorithms. Also, coexistent scheduling can be attempted in different types of FMS configurations with different sizes of FMS facilities, different numbers of jobs and MHRs, etc.

\section{Acknowledgment}

The authors would like to express sincere thanks to the editorial team and anonymous reviewers for their constructive comments on an earlier version of this research paper.

\section{References}

1. Mohapatra, P., Kumar, N., Matta, A., et al. "A nested partitioning-based approach to integrate process planning and scheduling in a flexible manufacturing environment", International Journal of Computer Integrated Manufacturing, 28(10), pp. 1077-1091 (2015).

2. Haq, A.N., Karthikeyan, T., and Dinesh, M. "Scheduling decisions in FMS using a heuristic approach", The International Journal of Advanced Manufacturing Technology, 22(5-6), pp. 374-379 (2003).

3. Chaudhry, I.A., Mahmood, S., and Shami, M. "Simultaneous scheduling of machines and automated guided vehicles in flexible manufacturing systems using genetic algorithms", Journal of the Central South University of Technology, 18(5), p. 1473 (2011).

4. Chawla, V.K., Chanda, A.K., Angra, S., et al. "Simultaneous dispatching and scheduling of multi-load AGVs in FMS-A simulation study", Materials Today: Proceedings, 5(11), pp. 25358-25367 (2018).

5. Chawla, V., Chanda, A., and Angra, S. "The scheduling of automatic guided vehicles for the workload balancing and travel time minimization in the flexible manufacturing system by the nature-inspired algorithm", Journal of Project Management, 4(1), pp. 1930 (2019).

6. Udhayakumar, P. and Kumanan, S. "Integrated scheduling of flexible manufacturing systems using evolutionary algorithms", The International Journal 
of Advanced Manufacturing Technology, 61(5-8), pp. 621-635 (2012).

7. Chawla, V.K., Chanda, A.K., and Angra, S. "Automatic guided vehicle systems in flexible manufacturing system-A review", International Journal of Industrial Engineering: Theory, Applications, and Practice, 26(5), pp. 737-765 (2019a).

8. Bozer, Y.A. and Srinivasan, M.M. "Tandem configurations for automated guided vehicle systems and the analysis of single-vehicle loops", IIE Transactions, 23(1), pp. $72-82$ (1991).

9. Bozer, Y.A. and Srinivasan, M.M. "Tandem AGV systems: A partitioning algorithm and performance comparison with conventional AGV systems", European Journal of Operational Research, 63(2), pp. 173191 (1992).

10. Angra, S., Chanda, A., and Chawla, V. "Comparison and evaluation of job selection dispatching rules for integrated scheduling of multi-load automatic guided vehicles serving in variable-sized flexible manufacturing system layouts: A simulation study", Management Science Letters, 8(4), pp. 187-200 (2018).

11. Chawla, V.K., Chanda, A.K., Angra, S., et al. "Effect of nature-inspired algorithms and hybrid dispatching rules on the performance of automatic guided vehicles in the flexible manufacturing system", Journal of the Brazilian Society of Mechanical Sciences and Engineering, 41(10), p. 391 (2019c).

12. Chawla, V., Angra, S., Suri, S., et al. "A synergic framework for cyber-physical production systems in the context of Industry 4.0 and beyond", International Journal of Data and Network Science, 4(2), pp. 237244 (2020).

13. Udhayakumar, P. and Kumanan, S. "Task scheduling of AGV in FMS using non-traditional optimization techniques", International Journal of Simulation Modelling, 9(1), pp. 28-39 (2010).

14. Chawla, V.K., Chanda, A., and Angra, S. "Sustainable multi-objective scheduling for automatic guided vehicles and flexible manufacturing system by a grey wolf optimization algorithm", International Journal of Data and Network Science, 2(1), pp. 27-40 (2018a).

15. Chawla, V., Chanda, A., and Angra, S. "Material handling robots fleet size optimization by a heuristic", Journal of Project Management, 4(3), pp. 177-184 (2019b).

16. Akturk, M.S. and Yilmaz, H. "Scheduling of automated guided vehicles in a decision-making hierarchy", International Journal of Production Research, 34(2), pp. 577-591 (1996).

17. Veeravalli, B., Rajesh, G., and Viswanadham, N. "Design and analysis of optimal material distribution policies in flexible manufacturing systems using a single AGV", International Journal of Production Research, 40(12), pp. 2937-2954 (2002).

18. Le-Anh, T. and De Koster, M.B.M. "A review of the design and control of automated guided vehicle systems", European Journal of Operational Research, 171(1), pp. 1-23 (2006).

19. Caumond, A., Lacomme, P., Moukrim, A., et al. "A MILP for scheduling problems in an FMS with one vehicle", European Journal of Operational Research, 199(3), pp. 706-722 (2009).

20. Dumas, Y., Desrosiers, J., and Soumis, F. "The pickup and delivery problem with time windows", European Journal of Operational Research, 54(1), pp. 7-22 (1991).

21. Bilge, Ü. and Ulusoy, G. "A time window approach to simultaneous scheduling of machines and material handling systems in an FMS", Operations Research, 43(6), pp. 1058-1070 (1995).

22. Rashidi, H. "Scheduling in container terminals using the network simplex algorithm", Journal of Optimization in Industrial Engineering, pp. 9-16 (2010).

23. Fazlollahtabar, H., Saidi-Mehrabad, M., and Balakrishnan, J. "Mathematical optimization for earliness/tardiness minimization in multiple automated guided vehicle manufacturing systems via integrated heuristic algorithms", Robotics and Autonomous Systems, 72, pp. 131-138 (2015).

24. Rashidi, H. and Tsang, E., Vehicle Scheduling in Port Automation: Advanced Algorithms for Minimum Cost Flow Problems, CRC Press (2015).

25. Jahromi, M.H.M.A., Tavakkoli-Moghaddam, R., Makui, A., et al. "A new mathematical model for a scheduling problem of dynamic machine-tool selection and operation allocation in a flexible manufacturing system: A modified evolutionary algorithm", Scientia Iranica, 24(2), pp. 765-777 (2017).

26. Rashidi Komijan, A., Tavakkoli-Moghaddam, R., and Dalil, S.A. "A mathematical model for an integrated airline fleet assignment and crew scheduling problem solved by vibration damping optimization", Scientia Iranica, 28(2), pp. 970-984 (2021).

27. Habibi, F., Barzinpour, F., and Sadjadi, S.J. "A mathematical model for project scheduling and material ordering problem with sustainability considerations: A case study in Iran", Computers \& Industrial Engineering, 128, pp. 690-710 (2019).

28. Sharma, N., Chawla, V., and Ram, N. "Comparison of machine learning algorithms for the automatic programming of a computer numerical control machine", International Journal of Data and Network Science, 4(1), pp. 1-14 (2020).

29. Savelsbergh, M.W. and Sol, M. "The general pickup and delivery problem", Transportation Science, 29(1), pp. 17-29 (1995).

30. Meersmans, P.J.M. "Optimization of container handling systems (No. 271)", Tinbergen Institute Research Series (2002).

31. Yang, J., Jaillet, P., and Mahmassani, H. "Real-time multivehicle truckload pickup and delivery problems", Transportation Science, 38(2), pp. 135-148 (2004). 
32. Ab Rashid, M.F.F., Tiwari, A., and Hutabarat, W. "Comparison of sequential and integrated optimization approaches for ASP and ALB", Procedia CIRP, 63, pp. $505-510$ (2017).

33. Chawla, V.K., Chanda, A.K., and Angra, S. "Multiload AGVs scheduling by application of modified memetic particle swarm optimization algorithm", Journal of the Brazilian Society of Mechanical Sciences and Engineering, 40(9), p. 436 (2018b).

34. Jerald, J., Asokan, P., Saravanan, R., et al. "Simultaneous scheduling of parts and automated guided vehicles in an FMS environment using an adaptive genetic algorithm", The International Journal of Advanced Manufacturing Technology, 29(5-6), pp. 584589 (2006).

35. Chanda, A., Angra, S., and Chawla, V. "A modified memetic particle swarm optimization algorithm for sustainable multi-objective scheduling of automatic guided vehicles in a flexible manufacturing system", International Journal of Computer-Aided Manufacturing, 4(1), pp. 33-47 (2018).

36. Buddala, R. and Mahapatra, S.S. "An integrated approach for scheduling flexible job-shop using teachinglearning-based optimization method", Journal of Industrial Engineering International, 15(1), pp. 181-192 (2019).

37. Corréa, A.I., Langevin, A., and Rousseau, L.M. "Scheduling and routing of automated guided vehicles: A hybrid approach", Computers \& Operations Research, 34(6), pp. 1688-1707 (2007).

38. Joshi, S. and Smith, J.S., Computer Control of Flexible Manufacturing Systems: Research and Development, Springer Science \& Business Media (2012).

39. Chawla, V.K., Chanda, A.K., and Angra, S. "Evaluation of tool selection rules in the flexible manufacturing system", International Journal of Industrial Engineering \& Production Research, 31(1), pp. 131142 (2020a).

40. Gamberi, M., Manzini, R., and Regattieri, A. "A new approach for the automatic analysis and control of material handling systems: integrated layout flow analysis (ILFA)", The International Journal of Advanced Manufacturing Technology, 41(1-2), p. 156 (2009).

41. Fazlollahtabar, H. and Saidi-Mehrabad, M. "Methodologies to optimize automated guided vehicle scheduling and routing problems: a review study", Journal of Intelligent \& Robotic Systems, 77(3-4), pp. 525-545 (2015).

42. Nishi, T., Hiranaka, Y., and Grossmann, I.E. "A bilevel decomposition algorithm for simultaneous production scheduling and conflict-free routing for automated guided vehicles", Computers \& Operations
Research, 38(5), pp. 876-888 (2011).

43. NageswaraRao, M., NarayanaRao, K., and Ranaga Janardhana, G. "Hybrid meta heuristic algorithm for simultaneous scheduling of machines and AGVs in flexible manufacturing environment", Canadian Journal of Basic and Applied Sciences, 3(02), pp. 29-44 (2015).

44. Umar, U.A., Ariffin, M.K.A., Ismail, N., et al. "Hybrid multi-objective genetic algorithms for integrated dynamic scheduling and routing of jobs and automatedguided vehicles (AGV) in flexible manufacturing systems (FMS) environment", The International Journal of Advanced Manufacturing Technology, 81(9-12), pp. 2123-2141 (2015).

45. Mallikarjuna, K., Veeranna, V., and Reddy, K.H. "A new meta-heuristics for optimum design of loop layout in a flexible manufacturing system with integrated scheduling", The International Journal of Advanced Manufacturing Technology, 84(9-12), pp. 1841-1860 (2016).

46. Zheng, Y., Xiao, Y., and Seo, Y. "A hybrid heuristic algorithm for the integrated problem of machine scheduling and unidirectional flow path design", International Journal of Industrial Engineering, 22(6) (2015).

47. Mirjalili, S., Mirjalili, S.M., Lewis, A., et al. "Advances in engineering software", Renewable and Sustainable Energy Reviews, pp. 46-61 (2014).

48. Mirjalili, S., Saremi, S., Mirjalili, S.M., et al. "Multiobjective grey wolf optimizer: a novel algorithm for multi-criterion optimization", Expert Systems with Applications, 47, pp. 106-119 (2016).

49. Bozorg-Haddad, O., Advanced Optimization by Nature-Inspired Algorithms, Singapore, Springer (2018).

50. Nawaz, M., Enscore Jr, E.E., and Ham, I. "A heuristic algorithm for the m-machine, n-job flow-shop sequencing problem", Omega, 11(1), pp. 91-95 (1983).

\section{Biographies}

V.K. Chawla, PhD, presently works as a faculty member in the Mechanical and Automation Engineering Department at Indira Gandhi Delhi Technical University for Women, Kashmiri Gate, Delhi. He earned his BTech degree in Mechanical Engineering from AKGEC, Ghaziabad U.P, MTech degree in Production Engineering from Delhi Institute of Tool Engineering, Delhi, and PhD from the National Institute of Technology Kurukshetra, Haryana. He has more than 11 years of experience in teaching, industry activities, research, and academic administration. His research interests include production and industrial engineering with focus on material handling systems in flexible manufacturing systems. He has more than 30 research publications in reputed national/international journals and conference proceedings. He is member of many professional bodies including the Institution of 
Engineers, the Society of Automotive Engineers, and ASME. He is also an editor of the International Journal of Data and Network Science, published by Growing Science, Canada.

Arindam Kumar Chanda received his BTech in Production Engineering from B.I.T. Sindri and ME and $\mathrm{PhD}$ in Production Engineering both from Jadavpur University, Kolkata. He has joined the Mechanical and Automation Engineering Department of G. B. Pant Government Engineering College in September, 2014. He has more than 21 years of total experience in the areas of teaching, research, industry activities, and management. He has served as Dean (Academics), HoD (MAE), First Appellate Authority, Chairman (Admission Committee), and Chairman/Member of several committees at GBPEC. Prior to joining GBPEC, Dr. Chanda had worked in different teaching positions at IGDTUW, Gautam Buddha University, NIFT and SMIT. His research/academic interests include production and industrial engineering, advanced manufacturing, robotics, and automation. Dr. Arindam Kumar Chanda also worked for 4 years as assistant manager in the Production Engineering Department of Heavy Engineering Industry and for 4 years as assistant director at All India Council for Technical Education. Prof. Chanda has guided 2 PhD scholars and evaluated several PhD and MTech theses at reputed Universities. He has published several research papers in peer reviewed and refereed journals and conferences of repute. Prof. Chanda has served as Expert Member in AICTE (EVC and SAC) and as Expert Member of Technical Committees both inside and outside GBPEC. He is reviewer in several indexed journals and has chaired sessions in conferences and seminars of repute. Dr. Arindam Kumar Chanda is the Fellow of the Institution of Engineers (India) and Life Member in several professional bodies like The Robotics Society, Indian Society for Technical Education, Indian Institution of Industrial Engineering, and Society of Automotive Engineers.

Surjit Angra is Professor and Former Head in the Department of Mechanical Engineering, National Institute of Technology Kurukshetra. He has been the director of All India Council for Technical Education, Ministry of HRD, Govt. of India. He has 36 years of experience in teaching, industry activities, and administration. He did his BSc in Mechanical Engineering at REC, Kurukshetra, in 1981. Also, he did his MTech in Mechanical Engineering in 1986 and PhD in 1995 at REC, Kurukshetra, presently known as NIT Kurukshetra. His area of interest is industrial tribology and machine design. He has supervised $10 \mathrm{PhD}$ and 52 MTech dissertations and theses. He has published more than 90 research papers in his area of research work in international journals like Tribology Letters, ASME, Tribology International, Journal of Reliability, Journal of System Safety, Journal of Engineering and Material Science, and Journal of IEI besides proceedings of ISME and so on.

Ali Bonyadi Naeini is a faculty member of the Business Department of Iran University of Science and Technology (IUST). He has taught several courses in the fields of marketing management, marketing research, and research design and method so far. He has also presented several conference papers and scholarly journal papers mostly focused on quantitative decisionmaking techniques. 